Pacific Journal of Mathematic 


\title{
ON THE COMPACTNESS OF THE HYPERSPACE OF FACES
}

\author{
H. B. Reiter and N. M. Stavrakas
}

Let $X \subset R^{d}, d>2$, be compact and convex. It is shown that the space of proper faces (poonems) of $X$ is compact if and only if the space of $k$-exposed (extreme) points of $X$ is compact, $0 \leqq k \leqq d-2$.

By a flat we mean a translate of a subspace of $R^{d}$ and by a hyperplane, a flat of dimension $d-1$. If $X$ is a compact convex subset of $R^{d}$ the symbols $\operatorname{dim}(X)$, rel int $(X)$, and rel bd $(X)$ denote respectively, the dimension of the flat generated by $X$, the interior, and the boundary of $X$ with respect to the flat $X$ generates. A hyperplane $H$ is called a supporting hyperplane of $X$ if $H \cap X \neq \varnothing$ and $H \cap \operatorname{relint}(X)=\varnothing$. A set $A$ is called a face of $X$ if $A=X$, $A=\varnothing$ or if there exists a supporting hyperplane $H$ of $X$ such that $A=H \cap X$. The set of proper faces (those not $X$ or $\varnothing$ ) is denoted by $\mathscr{F}(X) . \quad \mathrm{A}$ set $B$ is called a poonem of $X$ if there exists sets $B_{0}, B_{1}, \cdots, B_{m}$ such that $B_{m}=X$ and $B_{i-1} \in \mathscr{F}\left(B_{i}\right)$ for $i=1, \cdots, m$. The set of poonems of $X$ is denoted by $\mathscr{P}(X)$. A point $x$ in $X$ is called a $k$-exposed [ $k$-extreme] point if for some $j \leqq k, x$ belongs to a $j$-dimensional face [ $j$-dimensional poonem] of $X$. The symbols $\exp _{k}(X)$ and $\operatorname{ext}_{k}(X)$ denote the set of $k$-exposed and $k$-extreme points of $X$ respectively. A point $x$ of $X$ is called an exposed point of $X$ if $\{x\} \in \mathscr{F}(X)$ and $x$ is called an extreme point if whenever $x \in[a, b] \subset X$, we have $x=a$ or $x=b$, where $[a, b]$ denotes the closed line segment from $a$ to $b$. The symbols $\operatorname{ext}(X)$ and $\exp (X)$ denote the extreme points and exposed points of $X$, respectively. Note that $\exp (X)=\exp _{0}(X)$ and $\operatorname{ext}(X)=\operatorname{ext}_{0}(X)$. Also, let $q$ denote the Hausdorff metric. Finally, if $D \subset R^{d}$, the symbols $\operatorname{cl}(D)$ and conv $(D)$ denote the closure of $D$ and the convex hull of $D$ respectively.

We require the following results

Proposition. Let $X, A$ and $B$ be nonempty compact convex subsets of $R^{d}, d \geqq 2$, with $A \subset X$ and $B \subset X$.

(a) If $A, B \in F(X)(P(X))$ and $A \cap B \neq \varnothing$, then $A \cap B \in F(X)$ $(P(X))$.

(b) If $A \in F(X)(P(X))$ with relint $(B) \cap A \neq \varnothing$ then $B \subset A$.

(c) If $A \in F(X)(P(X))$ and $A \varsubsetneqq B \subset X$ then $A \in F(B)(P(B))$.

(d) $\operatorname{ext}_{k}(X) \subset \mathrm{cl}\left(\exp _{k}(X)\right)$.

(e) If $A \in F(X)$ then $\operatorname{ext}_{k}(A) \subset \operatorname{ext}_{k}(X)$. 
Propositions (b), (c) and (e) follow quickly from definitions while (a) is proved in Grunbaum [2]. Proposition (d) is due to Asplund [1]. The following theorem is the main communication of this note.

THEOREM. Let $X \subset R^{d}, d>2$, be compact and convex. Then

(i) The metric space $(P(X), q)$ is compact if and only if $\operatorname{ext}_{k}(X)$ is compact, $0 \leqq k \leqq d-2$.

(ii) The metric space $(F(X), q)$ is compact if and only if $\exp _{k}(X)$ is compact, $0 \leqq k \leqq d-2$.

Proof. We consider part (i). The only if part is obvious. Suppose $\left\{A_{n}\right\} \rightarrow A$ with $A_{n} \in P(X)$ for all $n$. Clearly if $\operatorname{dim}(A)=0, A$ is a limit point of $\operatorname{ext}(X)$ and we are done. We proceed now by induction on the dimension of $A$. Let $\operatorname{dim}(A)=k+1$. We may suppose $k+1 \leqq d-2$ since if $k+1=d-1$ the sequence $\left\{A_{n}\right\}$ is eventually constant and we are done. We consider cases:

Case 1. For infinitely many $n, \operatorname{dim}\left(A_{n}\right)=k+1$. Without loss of generality we may suppose $\operatorname{dim}\left(A_{n}\right)=k+1$ (otherwise we pass to an appropriate subsequence). Let $x \in \operatorname{relint}(A)$. Since $x$ is a limit point of the set $\operatorname{ext}_{k \perp 1}(X)$ and $k+1 \leqq d-2$ our hypotheses imply that there exists $Q \in P(X)$ with $\operatorname{dim}(Q)=k+1$ and $x \in Q$. By Proposition (b) $A \subset Q$. We claim $A=Q$. Suppose not. Then there exists $y \in \operatorname{rel} b d(A) \cap \operatorname{relint}(Q)$ and there exists a sequence $\left\{y_{n}\right\} \rightarrow y$ with $y_{n} \in \operatorname{rel~bd}\left(A_{n}\right)$ for all $n$. Let $F_{n} \in P\left(A_{n}\right)$ with $y_{n} \in F_{n}$ and $F_{n} \neq A_{n}$ for all $n$. Without loss of generality we may suppose $\left\{F_{n}\right\} \rightarrow F$ for some $F$. Since $\operatorname{dim}\left(F_{n}\right) \leqq k$ for all $n$ we have $\operatorname{dim}(F) \leqq k$. Since we have that $F_{n} \in P(X)$ for all $n$ and since $\operatorname{dim}(F) \leqq k$ the induction hypothesis implies $F \in P(X)$. Since $F \cap \operatorname{rel} \operatorname{int}(Q) \neq \varnothing$, Proposition (b) implies $Q \subset F$, a contradiction since $\operatorname{dim}(F)<\operatorname{dim}(Q)$. Thus $A=Q$ and we are done.

Case 2. There is no subsequence of $\left\{A_{n}\right\}$ each element of which has dimension $k+1$. Then there exists $\left\{x_{n}\right\} \rightarrow x$ with $x_{n} \in \operatorname{rel}$ bd $\left(A_{n}\right)$ for all $n$. Let $F_{n} \in P\left(A_{n}\right), F_{n} \neq A_{n}$, with $x_{n} \in F_{n}$ for all $n$. Without loss of generality we may suppose $\left\{F_{n}\right\} \rightarrow F$ for some $F$. Note $F_{n} \in$ $P(X)$ and $\operatorname{dim}\left(F_{n}\right)<\operatorname{dim}\left(A_{n}\right)$ for all $n$. If there is a subsequence of $\left\{F_{n}\right\}$ each element of which has dimension $k+1$ then $x$ is a limit point of $\operatorname{ext}_{k+1}(X)$ and we have returned to the argument of the first case. If not, we repeat the latter procedure as many times as needed to return.

We turn now to the proof of part (ii). The only if part is trivial and is omitted. In view of (i), we need only show "a face of a face is a face." Let $A \in \mathscr{F}(X), \operatorname{dim}(A) \geqq 1$ and let $B \in \mathscr{F}(A)$ and sup- 
pose $\operatorname{dim}(B)=k$. We may suppose $B$ is a maximal proper face of $A$ with respect to set inclusion. By definition $B \subset \operatorname{ext}_{k}(X)$ and by hypothesis and Propositions (e) and (d) $B \subset \exp _{k}(X)$. Let $x \in \operatorname{rel} \operatorname{int}(B)$ and let $Q \in \mathscr{F}(X)$ with $x \in Q$ and $\operatorname{dim}(Q)=j \leqq k$. Since $\operatorname{rel} \operatorname{int}(B) \cup$ $(A \cap Q) \neq \varnothing$, by Propositions (a) and (b), $B \subset A \cap Q$. Since $\operatorname{dim}(A \cap Q) \leqq$ $\operatorname{dim}(Q) \leqq j \leqq k<\operatorname{dim}(A)$, we have $A \cap Q \neq A$. Since we have that $A \cap Q \in \mathscr{F}(X)$ Proposition (c) implies that $A \cap Q \in \mathscr{F}(A)$. We must now have $B=A \cap Q$ since $B$ is a maximal proper face of $A$. This completes the proof of (ii).

If one defines $f: \operatorname{rel} b d(X) \rightarrow P(X)$ where $f(x)$ is the smallest poonem of $X$ containing $x$, then one has an example of the face function introduced by V. Klee and M. Martin [3]. Part (i) provides a characterization of those compact convex sets $X$ in $R^{d}$ for which $f$ is continuous at each point of $\operatorname{rel~bd}(X)$.

We wish to consider some examples from $R^{3}$ and $R^{4}$. Let $D$ be a compact convex subset of $R^{3}$ with nonempty interior such that $\exp (D)$ is compact but $(\mathscr{F}(D), q)$ is not compact. It is not difficult to show that there is a 1-poonem $B$ of $D$ which is not a face of $D$. On the basis of the latter example, one might conjecture that for a compact subset $X$ of $R^{3}(\mathscr{F}(X), q)$ is compact if and only if $\exp (X)$ is compact and for each $A \in \mathscr{F}(X), \mathscr{F}(A) \subset \mathscr{F}(X)$ i.e., "a face of a face is a face." While this is true in $R^{3}$ it is not in $R^{4}$ as the following example shows.

Let $C=\left\{(x, y) \mid x^{2}+y^{2}=1, x \geqq 0, y \geqq 0\right\}$ and let $E=\operatorname{conv}\{(1,0)$, $(-1,0),(0,1),(0,-1)\}$. Let $l$ be the closed line segment from $(0,-1)$ to $(0,1)$ in $E$. Let $\left\{x_{n}\right\}$ be a sequence of distinct points in $C$ convergent to $(1,0)$. Regarding $R^{4}$ as $R^{2} \times R^{2}$ let $F_{i}=\left\{x_{i}\right\} \times l$ for $i=$ $1,2, \cdots$ and let $F_{0}=\{(1,0)\} \times E$. Define $X$ as $X=\operatorname{conv}\left(\bigcup_{i=0}^{\infty} F_{i}\right)$. One may check that $\exp (X)$ is compact and that "a face of a face is a face." Note also for each $i, F_{i} \in \mathscr{F}(X)$ and $\left\{F_{i}\right\} \rightarrow F$ where $F=\{(1,0)\} \times l$. However, $F \notin \mathscr{F}(X)$ so that $(\mathscr{F}(X), q)$ is not compact. (This particular example was pointed out by V. Klee; the authors had a more cumbersome one.) Returning to the set $D$ in $R^{3}$ mentioned earlier, perhaps the more important observation about $D$ (as far as higher dimensions are concerned) is not that we have $B \in \mathscr{P}(D)$ and $B \notin \mathscr{F}(D)$ but rather that $\exp _{1}(D)$ is not compact since the theorem in $R^{3}$ that generalizes is that $(\mathscr{F}(X), q)$ is compact if and only if $\exp (X)$ and $\exp _{1}(X)$ are compact.

\section{REFERENCES}

1. E. Asplund, A k-extreme point is the limit of $k$ exposed points, Israel J. Math., $\mathbf{1}$ (1963), 161-162.

2. B. Grunbaum, Convex Polytopes, John Wiley and Sons, New York, 1967. 
3. V. Klee and M. Martin, Semicontinuity of the face function of a convex set, Commetarii Mato Helvetrci, 46 (1971), 1-12.

Received March 1, 1976 and in revised form April 20, 1977.

UNIVERSITY OF NORTH CAROLINA

Charlotte, NC 28223 


\section{PACIFIC JOURNAL OF MATHEMATICS}

EDITORS

RICHARD ARENS (Managing Editor)

University of California

Los Angeles, California 90024

C. W. Curtis

University of Oregon

Eugene, OR 97403

C. C. MOORE

J. DUGUNDJI

Department of Mathematics University of Southern Californa Los Angeles, California 90007

R. Finn AND J. Milgram Stanford University Stanford, California 94305

University of California

Berkeley, CA 94720

\section{ASSOCIATE EDITORS}

E. F. BeCKENBACH

B. H. NeUMANN

F. WOLF

K. YosHida

\section{SUPPORTING INSTITUTIONS}

UNIVERSITY OF BRITISH COLUMBIA UNIVERSITY OF SOUTHERN CALIFORNIA CALIFORNIA INSTITUTE OF TECHNOLOGY UNIVERSITY OF CALIFORNIA MONTANA STATE UNIVERSITY UNIVERSITY OF NEVADA, RENO STANFORD UNIVERSITY UNIVERSITY OF TOKYO UNIVERSITY OF UTAH NEW MEXICO STATE UNIVERSITY WASHINGTON STATE UNIVERSITY OREGON STATE UNIVERSITY UNIVERSITY OF WASHINGTON UNIVERSITY OF OREGON OSAKA UNIVERSITY 


\section{Pacific Journal of Mathematics \\ Vol. 73, No. 1 \\ March, 1977}

Thomas Robert Berger, Hall-Higman type theorems. $V \ldots \ldots \ldots \ldots \ldots \ldots \ldots$

Frank Peter Anthony Cass and Billy E. Rhoades, Mercerian theorems via

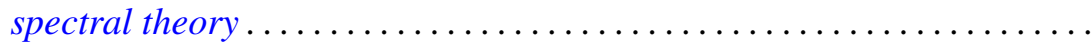

Morris Leroy Eaton and Michael David Perlman, Generating $\mathrm{O}(n)$ with

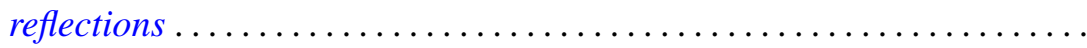

Frank John Forelli, Jr., A necessary condition on the extreme points of a

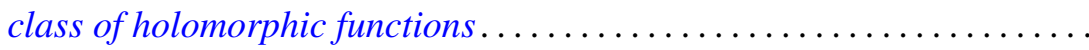

Melvin F. Janowitz, Complemented congruences on complemented

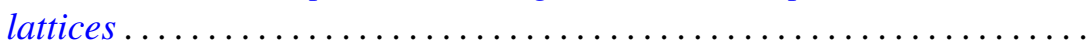

Maria M. Klawe, Semidirect product of semigroups in relation to amenability, cancellation properties, and strong $F \phi$ lner conditions....

Theodore Willis Laetsch, Normal cones, barrier cones, and the "spherical image" of convex surfaces in locally convex spaces ................

Chao-Chu Liang, Involutions fixing codimension two knots.............

Joyce Longman, On generalizations of alternative algebras .............

Giancarlo Mauceri, Square integrable representations and the Fourier

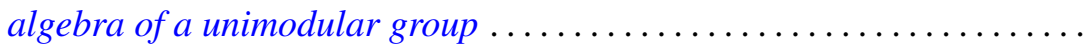

J. Marshall Osborn, Lie algebras with descending chain condition...

John Robert Quine, Jr., Tangent winding numbers and branched

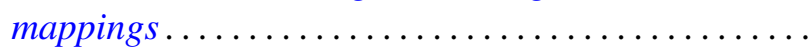

Louis Jackson Ratliff, Jr. and David Eugene Rush, Notes on ideal covers and associated primes .

H. B. Reiter and N. Stavrakas, On the compactness of the hyperspace of faces.

Walter Roth, A general Rudin-Carlson theorem in Banach-spaces ..

Mark Andrew Smith, Products of Banach spaces that are uniformly rotund in every direction.

Roger R. Smith, The R-Borel structure on a Choquet simplex ...

Gerald Stoller, The convergence-preserving rearrangements of real infinite series. ...

Graham H. Toomer, Generalized homotopy excision theorems modulo a Serre class of nilpotent groups...

Norris Freeman Weaver, Dehn's construction and the Poincaré conjecture....

Steven Howard Weintraub, Topological realization of equivariant intersection forms... 